\title{
Hydrocephalus: Ventricular Volume Quantification Using Three-Dimensional Brain CT Data and Semiautomatic Three-Dimensional Threshold-Based Segmentation Approach
}

\author{
Hyun Woo Goo, MD, PhD \\ Department of Radiology and Research Institute of Radiology, Asan Medical Center, University of Ulsan College of Medicine, Seoul, Korea
}

Objective: To evaluate the usefulness of the ventricular volume percentage quantified using three-dimensional (3D) brain computed tomography (CT) data for interpreting serial changes in hydrocephalus.

Materials and Methods: Intracranial and ventricular volumes were quantified using the semiautomatic 3D thresholdbased segmentation approach for 113 brain CT examinations (age at brain CT examination $\leq 18$ years) in 38 patients with hydrocephalus. Changes in ventricular volume percentage were calculated using 75 serial brain CT pairs (time interval $173.6 \pm$ 234.9 days) and compared with the conventional assessment of changes in hydrocephalus (increased, unchanged, or decreased). A cut-off value for the diagnosis of no change in hydrocephalus was calculated using receiver operating characteristic curve analysis. The reproducibility of the volumetric measurements was assessed using the intraclass correlation coefficient on a subset of 20 brain CT examinations.

Results: Mean intracranial volume, ventricular volume, and ventricular volume percentage were $1284.6 \pm 297.1 \mathrm{~cm}^{3}, 249.0 \pm$ $150.8 \mathrm{~cm}^{3}$, and $19.9 \pm 12.8 \%$, respectively. The volumetric measurements were highly reproducible (intraclass correlation coefficient $=1.0)$. Serial changes $(0.8 \pm 0.6 \%)$ in ventricular volume percentage in the unchanged group $(n=28)$ were significantly smaller than those in the increased and decreased groups $(6.8 \pm 4.3 \%$ and $5.6 \pm 4.2 \%$, respectively; $p=0.001$ and $p<0.001$, respectively; $\mathrm{n}=11$ and $\mathrm{n}=36$, respectively). The ventricular volume percentage was an excellent parameter for evaluating the degree of hydrocephalus (area under the receiver operating characteristic curve $=0.975 ; 95 \%$ confidence interval, $0.948-1.000 ; p<0.001$ ). With a cut-off value of $2.4 \%$, the diagnosis of unchanged hydrocephalus could be made with $83.0 \%$ sensitivity and $100.0 \%$ specificity.

Conclusion: The ventricular volume percentage quantified using 3D brain CT data is useful for interpreting serial changes in hydrocephalus.

Keywords: Brain ventricular system; Computed tomography; Children; Hydrocephalus; Image segmentation; Volume measurement

\section{INTRODUCTION}

Brain computed tomography (CT) is widely used to monitor the degree of hydrocephalus because it can be performed quickly with a reduced radiation dose using an optimized protocol, and it provides an isotropic high spatial resolution that enables high-quality threedimensional (3D) visualization and segmentation $(1,2)$. It is challenging for radiologists to accurately evaluate subtle changes in hydrocephalus by only visually assessing serial

Received: March 31, 2020 Revised: June 15, 2020 Accepted: June 22, 2020

Corresponding author: Hyun Woo Goo, MD, PhD, Department of Radiology and Research Institute of Radiology, Asan Medical Center, University of Ulsan College of Medicine, 88 Olympic-ro 43-gil, Songpa-gu, Seoul 05505, Korea.

- E-mail: ghw68@hanmail.net

This is an 0pen Access article distributed under the terms of the Creative Commons Attribution Non-Commercial License (https:// creativecommons.org/licenses/by-nc/4.0) which permits unrestricted non-commercial use, distribution, and reproduction in any medium, provided the original work is properly cited. 
brain CT scans, as head positions are often not identical due to differences introduced by head tilting and rotation between examinations of the same patient $(3,4)$. To avoid substantial delays in clinical decision making in urgent situations, various linear indices of ventricular volume, such as Evans index and fronto-occipital horn ratio, have been used in children with hydrocephalus $(5,6)$. Because subtle changes are difficult to determine using the linear indices or subjective assessments, ventricular volume quantified using brain $\mathrm{CT}$ data may be useful for the accurate detection of serial changes in hydrocephalus by radiologists (3). The 3D quantification of the intracranial ventricular volume using brain $\mathrm{CT}$ data and the thresholding technique and a region-growing algorithm has been attempted (3, 7-9). The semiautomatic 3D threshold-based segmentation approach was successfully implemented for volumetric quantification of cardiac chambers using cardiac CT data in our institution $(10,11)$, and based on the experience, we applied the same approach to the quantification of intracranial ventricular volume using brain $\mathrm{CT}$ data in patients with hydrocephalus. Therefore, the purpose of this retrospective study was to evaluate the usefulness of 3D quantified ventricular volume percentage based on brain $\mathrm{CT}$ for interpreting serial changes in hydrocephalus.

\section{MATERIALS AND METHODS}

This retrospective study was approved by the Institutional Review Board of our institution. The requirement for informed consent was waived (IRB No. 2020-0255).

\section{Study Population}

Between 2013 and 2016, ventricular volumes were quantified in 140 unenhanced brain CT examinations of 65 patients ( $\leq 18$ years of age) with hydrocephalus. Patients aged $>18$ years $(n=0)$ and those who underwent only one unenhanced brain CT examination $(n=27)$ were excluded from this study. The study involved 113 unenhanced brain CT examinations in 38 consecutive patients (18 males and 20 females; age at brain CT examination $3.7 \pm 4.9$ years, range 25 days -18 years) with hydrocephalus who underwent at least two serial brain CT examinations. Of the 113 brain $\mathrm{CT}$ examinations, there were 75 pairs of serial CT examinations in the same patients (time interval $173.6 \pm 234.9$ days). Etiologies of hydrocephalus included intraventricular hemorrhage $(n=13)$, tumor or cyst $(n=$ 11), idiopathic aqueductal or foramen Monro stenosis $(n=9)$, skeletal dysplasia involving the skull $(n=3)$, and extraventricular hydrocephalus $(n=2)$.

\section{Unenhanced Brain CT}

Spiral brain CT scanning was acquired with a 64-detectorrow CT scanner (SOMATOM Definition AS plus, Flash, or Edge; Siemens Healthineers). Spiral scan parameters were as follows: beam collimation $=64 \times 0.6 \mathrm{~mm}$, gantry rotation time $=0.28,0.33,0.5$, or $1.0 \mathrm{~s}$, pitch $=1.0$, thin axial slice width $=0.75 \mathrm{~mm}$, thin axial reconstruction interval $=0.4$ $\mathrm{mm}$, and thick multiplanar (axial, coronal and sagittal) slice thickness $=3,4$, or $5 \mathrm{~mm}$. The volume CT dose index based on a $16-\mathrm{cm}$ phantom $(23.8 \pm 6.4 \mathrm{mGy})$ was used to adapt the radiation dose of brain $\mathrm{CT}$ to the patient body weight $(12,13)$. Variable tube voltages, including $80 \mathrm{kV}(\mathrm{n}=54)$, $100 \mathrm{kV}(\mathrm{n}=24), 120 \mathrm{kV}(\mathrm{n}=30)$, and $140 \mathrm{kV}(\mathrm{n}=5)$ were used, and tube current was individually adjusted according to the body weight-based volume CT dose index values. An attenuation-based tube current modulation (CARE Dose 4D; Siemens Healthineers) was applied to reduce tube current while maintaining image noise in all brain $\mathrm{CT}$ examinations (14). In addition, organ-based tube current modulation (X-CARE; Siemens Healthineers) was used to reduce the dose to the eyes during CT scanning (15). All brain CT images were reconstructed using an iterative reconstruction algorithm (sinogram-affirmed iterative reconstruction; Siemens Healthineers) with a strength of 5 to reduce image noise $(16,17)$. The mean dose length product of brain CT examinations was $399.3 \pm 146.8 \mathrm{mGy} \cdot \mathrm{cm}$, and the mean dose length product-based effective dose estimate using age- and sex-specific conversion factors (13) of brain CT examination was $1.3 \pm 0.3 \mathrm{mSv}$.

\section{Quantification of Intracranial and Ventricular Volumes}

The stacks of thin axial brain CT images were sent to a commercially available workstation (Advantage Windows 4.6; GE Healthcare) for volume quantification. To obtain intracranial volume (Fig. 1), a 3D thresholdbased segmentation (lower threshold 0.0 Hounsfield units [HU], upper threshold $53.3 \pm 4.7 \mathrm{HU}$ ) was initially applied, followed by 3D erosion, 3D dilation, 3D region growing, and 3D hole filling. Similarly, a 3D thresholdbased segmentation approach (lower threshold $-2.5 \pm$ 3.9 HU, upper threshold $19.0 \pm 2.5 \mathrm{HU}$ ) was used to separate the intracranial cerebrospinal fluid from the brain, and extraventricular cerebrospinal fluid adjacent to the ventricles, most commonly the basal cistern, was 
eliminated manually to obtain the volume of the whole ventricular system including both lateral, third, and fourth ventricles (Fig. 2). Preliminary processing was conducted by a radiology technologist who had been working in a 3D image laboratory in our institution for more than 10 years, and the processed results were interactively reviewed on a $3 \mathrm{D}$ workstation and approved by an experienced pediatric radiologist (20-year experience in pediatric neuroimaging). The processing time required for the volume quantification was approximately 10-15 minutes for each CT examination, which was spent mainly on the manual adjustment of the segmented ventricles.

Small residual hyperdense intraventricular hematomas were observed in six brain CT examinations in two patients (acute hematoma that developed after the placement of a ventriculoperitoneal shunt in one patient and chronic calcified hematomas in the other patient). These hematomas were included for quantifying intracranial volume, but not

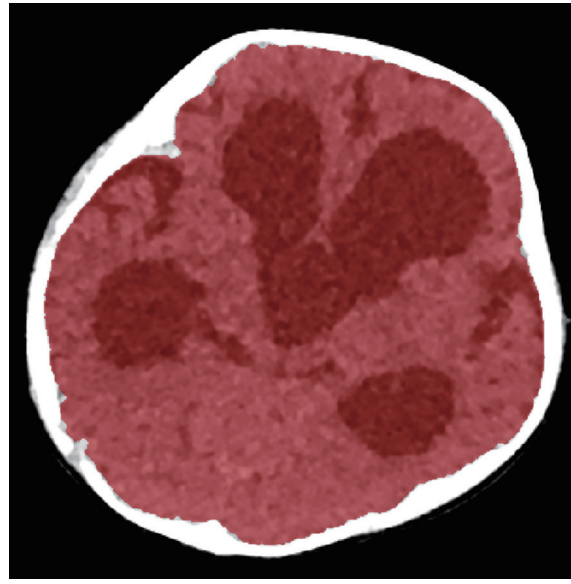

A

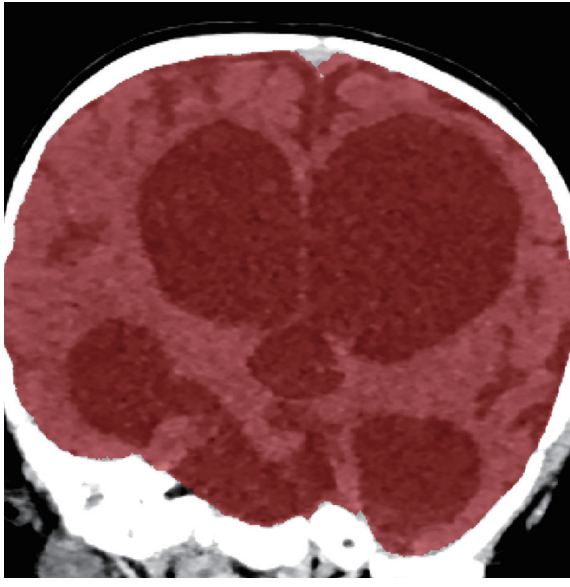

B

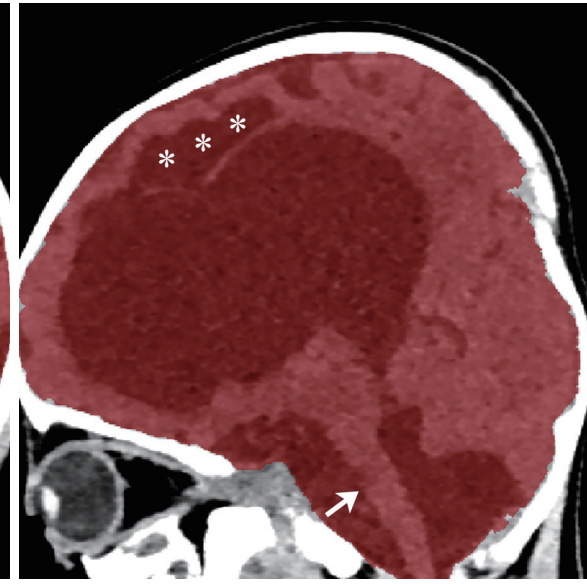

C

Fig. 1. An 8-month-old girl with posthemorrhagic hydrocephalus.

Axial (A), coronal (B), and sagittal (C) brain CT images demonstrate a segmented intracranial space (red layer) using a three-dimensional threshold-based approach. The lateral and third ventricles are severely dilated, which suggests obstruction at the aqueductal level. Cystic encephalomalacia (asterisks) and brain stem atrophy (arrow) due to a concomitant hypoxic ischemic brain injury are noted (C). CT = computed tomography
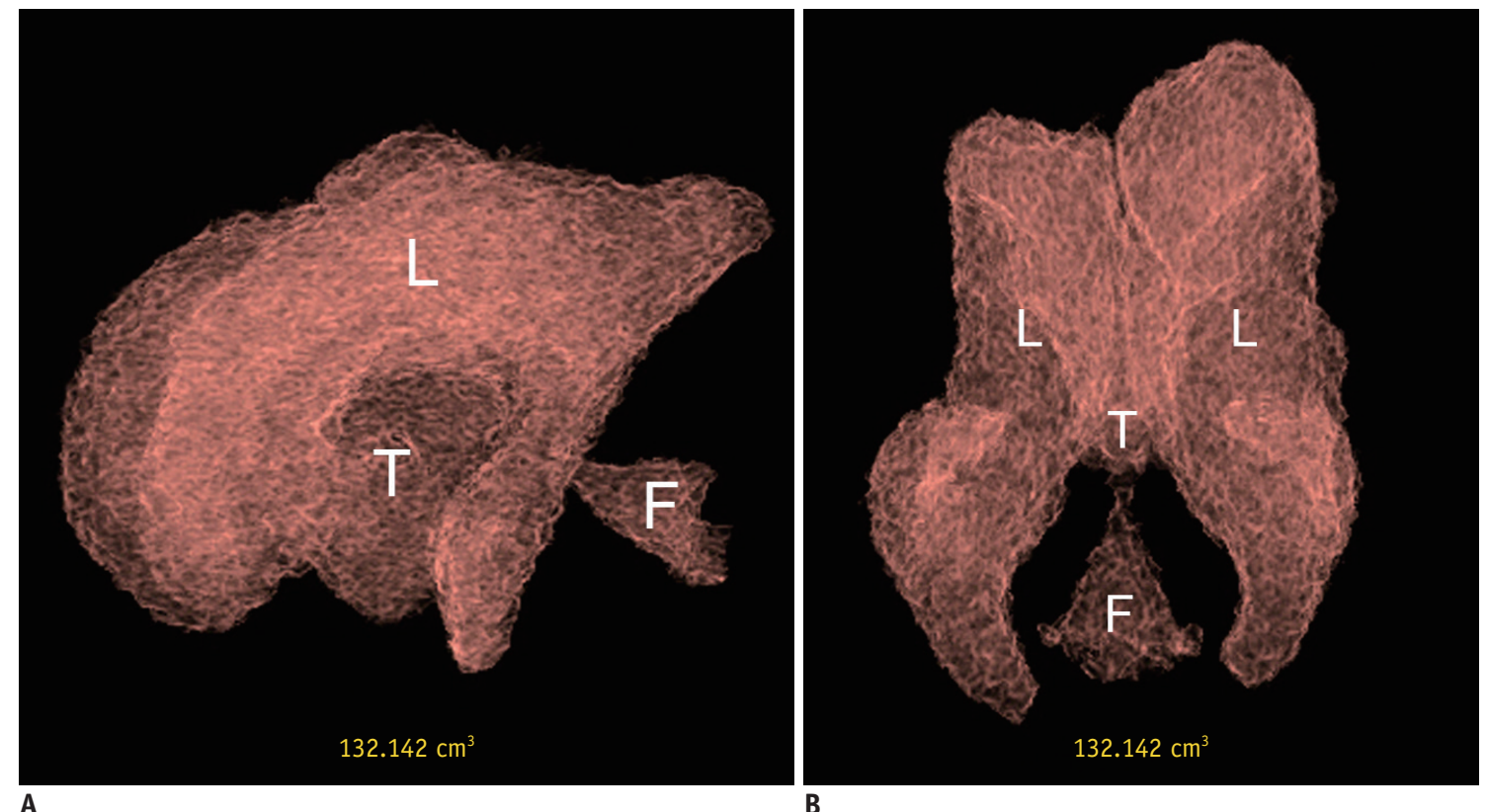

Fig. 2. A 1-year-old girl with choroid plexus papilloma and obstructive hydrocephalus.

Lateral (A) and frontal (B) volume-rendered brain CT images show the three-dimensional volume of the intracranial ventricles segmented using the semiautomatic three-dimensional threshold-based approach. $F=$ fourth ventricle, $L=$ lateral ventricle, $T=$ third ventricle 
for ventricular volume, because their attenuation values are substantially higher than those of the cerebrospinal fluid in the ventricles.

\section{Reproducibility of Intracranial and Ventricular Volume Measurements}

To evaluate the reproducibility of the volumetric measurements, the intracranial and ventricular volumes were measured twice in a subset of 20 brain $\mathrm{CT}$ examinations. The second session of the measurements was performed 3 months after the first session. Inter-measurement variability was represented as the mean difference percentage and the intraclass correlation coefficient of the two measurements.

\section{Serial Changes in Hydrocephalus}

The ventricular volume percentage was calculated as ventricular volume divided by intracranial volume for each brain CT examination. Subsequently, serial changes in hydrocephalus were evaluated in 75 serial brain CT pairs of the same patients by using ventricular volume percentage changes and conventional assessments (3 groups: increased, unchanged, or decreased) in official brain CT reports by the experienced pediatric radiologist (20-year experience in pediatric neuroimaging) who was blinded to the results of the ventricular volume percentages. For conventional assessment, the maximal transverse diameter of the third ventricle, the degree of blunting of the ventricular horns, and the degree of thinning of the cerebral mantle were collectively evaluated. This radiologic interpretation based on visual perception and two-dimensional measurements was regarded as the reference because it is the best among currently available evaluations. The absolute values of ventricular volume percentage changes of the three conventional assessment groups were compared.

To assess the diagnostic value of the ventricular volume percentage changes in evaluating serial changes in hydrocephalus, the area under the receiver operating characteristic curve was calculated. In addition, a cut-off value for the diagnosis of no change in hydrocephalus was calculated using the receiver operating characteristic curve analysis to evaluate its diagnostic performance.

The CT pairs with low diagnostic confidence for conventional assessment (i.e., suspicious serial change of one category, or calling two categories simultaneously, such as no change or slight interval decrease) based on the official reports were recorded. Furthermore, changes in the conventional interpretations of the CT pairs with low diagnostic confidence after applying the optimal cut-off value were evaluated.

\section{Statistical Analysis}

Continuous variables were expressed as mean \pm standard deviation or median with range. The unpaired $t$ test was used to compare the means of the two independent groups. Receiver operating characteristic curve analysis was used to assess the diagnostic ability of the binary classifier system (change or no change in the degree of hydrocephalus on serial brain CT examination in this study). Statistical analyses were performed using SPSS version 24.0 (IBM Corp.). A $p$ value of $\leq 0.05$ was considered statistically significant.

\section{RESULTS}

Mean intracranial volume, ventricular volume, and ventricular volume percentage were $1284.6 \pm 297.1 \mathrm{~cm}^{3}$, $249.0 \pm 150.8 \mathrm{~cm}^{3}$, and $19.9 \pm 12.8 \%$, respectively. In the subset of 20 brain CT examinations, the two measurements of intracranial volume were $1190.8 \pm 368.5$ $\mathrm{cm}^{3}$ and $1191.6 \pm 368.0 \mathrm{~cm}^{3}$, respectively, with a mean difference of $0.2 \pm 0.3 \%$, and the two measurements of ventricular volume were $201.2 \pm 125.0 \mathrm{~cm}^{3}$ and $201.2 \pm$ $125.1 \mathrm{~cm}^{3}$, respectively, with a mean difference of $0.5 \pm$ $0.3 \%$. In addition, the volumetric measurements were highly reproducible (intraclass correlation coefficient $=1.0$ for both intracranial and ventricular volumes, $p$ values $<0.001$ ).

Serial changes $(0.8 \pm 0.6 \%)$ in ventricular volume percentage in the unchanged group $(n=28)$ were significantly smaller than those in the increased and decreased groups $(6.8 \pm 4.3 \%$ and $5.6 \pm 4.2 \%$, respectively; $p=0.001$ and $p<0.001$, respectively; $\mathrm{n}=11$ and $\mathrm{n}=36$, respectively). The ventricular volume percentage showed an excellent diagnostic ability for evaluating the degree of hydrocephalus (area under the receiver operating characteristic curve $=0.975 ; 95 \%$ confidence interval, $0.948-1.000 ; p<0.001$ ) (Fig. 3). With a cut-off value of $2.4 \%$, the diagnosis of unchanged hydrocephalus could be made with $83.0 \%$ sensitivity and $100.0 \%$ specificity.

Low diagnostic confidence was observed in $13.3 \%$ (10/75) of the serial brain CT pairs, and their mean serial change in ventricular volume percentage was $2.7 \pm 1.5 \%$. When the optimal cut-off value of $2.4 \%$ was applied, the interpretation of two of the pairs changed from decreased hydrocephalus to unchanged hydrocephalus; the 


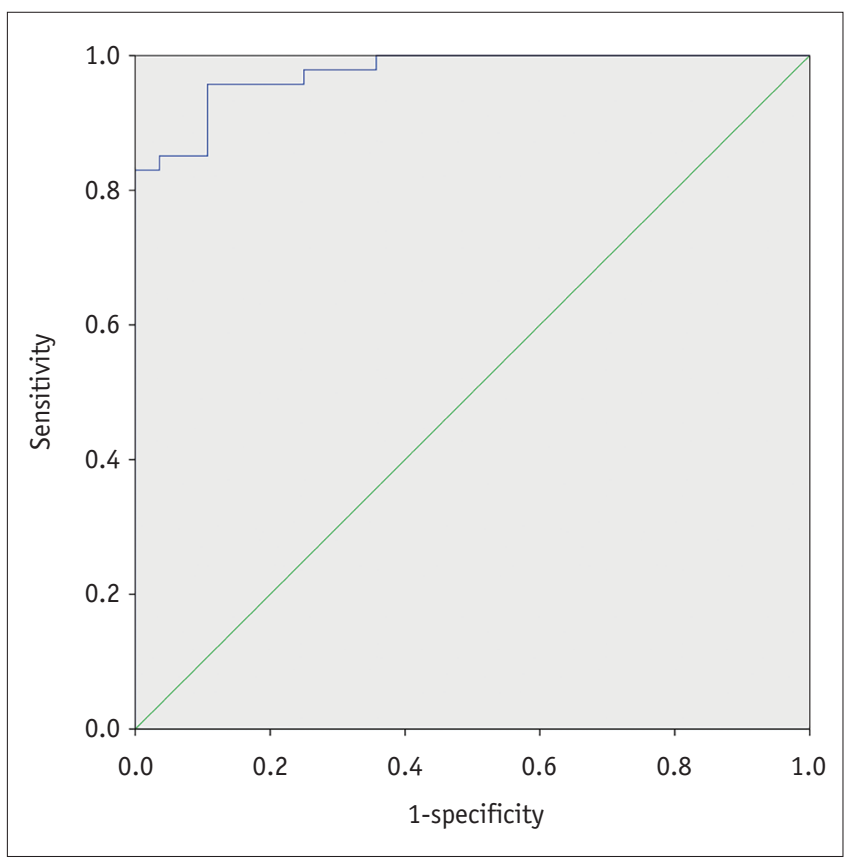

Fig. 3. Receiver operating characteristic curve demonstrating the excellent diagnostic ability of serial changes in ventricular volume percentage on serial brain CT examinations for evaluating the degree of hydrocephalus (area under the receiver operating characteristic curve $=0.975 ; 95 \%$ confidence interval, $0.948-1.000 ; p<0.001)$.

interpretation of the other eight pairs remained unchanged.

\section{DISCUSSION}

The ventricular volume percentages quantified using 3D brain CT data and the semiautomatic 3D threshold-based segmentation approach demonstrated high reproducibility and diagnostic ability for unchanged hydrocephalus. The greatest clinical implication of this study is that ventricular volume quantification may aid radiologists in interpreting changes in hydrocephalus on serial brain CT examinations. The quantitative evaluation showed the potential to improve diagnostic confidence in $20 \%(2 / 10)$ of the cases in this study. As previously mentioned, conventional visual assessment of subtle changes in hydrocephalus on brain $\mathrm{CT}$ is cumbersome, and this limits diagnostic accuracy. of interest, a recent study demonstrated that an automated CT registration and subtraction tool improved the reader's ability to detect subtle changes in hydrocephalus (4). If serial changes in ventricular volume percentage can be accessed during the interpretation of brain CT of patients with hydrocephalus, it will facilitate more accurate detections of serial changes in hydrocephalus, especially for inexperienced readers.
The fourth ventricle was excluded from the quantification of the ventricular system in a few studies $(9,18)$. However, the fourth ventricle is also enlarged in extraventricular hydrocephalus, and an isolated fourth ventricle enlargement is a rare complication of neurosurgical treatment.

Consequently, it is important to include the fourth ventricle in the intracranial ventricle segmentation as performed in this study.

In urgent situations, the 10-15 minutes required for the semiautomatic 3D threshold-based segmentation of intracranial and ventricular volumes may be considered too long by clinicians and radiologists. The processing time was spent mainly on manual elimination of the cerebral cisterns from the segmented intracranial cerebrospinal fluid. In a previous study (3), the volume of interest delimited by an elliptically shaped region of interest was used to exclude as much of the subarachnoid space as possible and shorten the processing time (mean: 2 minutes 42 seconds). However, the inclusion of cerebral cisterns adjacent to the ventricles, such as the basal cistern, was unavoidable, and this resulted in an overestimation of ventricular volume (3). A few recent studies demonstrated that automated intracranial ventricle segmentation software using deep learning methods could reduce processing time (e.g., to less than 1 minute) with high accuracy $(18,19)$. However, these automatic tools produced segmentation errors and remained at a pre-clinical stage of research $(18,19)$. The accuracy of deep learning methods depends on the quality and the number of ground truth data used for training. The segmented data obtained in this study may be used as ground truth in future studies using deep learning methods.

of importance, intracranial space volume demonstrated a progressive increase with age $(7,20)$. Therefore, the ventricular volume percentage may be better for the accurate and reproducible evaluation of serial changes in hydrocephalus as in this study. In contrast, the use of only serial changes in ventricular or cerebrospinal fluid volume, as in previous studies (7-9), may be misleading in evaluating the degree of hydrocephalus. In addition to volume changes, we should consider intracranial pressure changes in patients with hydrocephalus. CT-measured volume percentages appear more useful in patients with decreased ventricular compliance because small volume changes may cause large pressure increases (3).

Intracranial volume may not reflect actual brain volume in cases of enlarged subarachnoid space or brain parenchymal loss. In this regard, 3D volumetric measurements of the 
brain would reflect the degree of brain development or function better than intracranial volume. The fast brain magnetic resonance imaging protocol comprising T2weighted imaging sequences showed a good result even without sedation and ionizing radiation for routine surveillance imaging in pediatric hydrocephalus (21). We also plan to create a fast 3D brain magnetic resonance imaging protocol for evaluating hydrocephalus.

According to age- and gender-specific estimates of cumulative CT dose over 5 years using real radiation dose tracking data, the high frequency of brain CT scans was closely related to greater cumulative CT dose estimates in children with hydrocephalus (22). As a result, the radiation dose during each brain CT examination should be minimized for these patients. In this study, mean effective dose estimates of brain $\mathrm{CT}$ examinations could be reduced to $1.3 \mathrm{mSv}$. The main techniques applied for radiation dose reduction were body weight-adjusted protocol, attenuationbased tube current modulation, and the iterative reconstruction algorithm. Furthermore, the radiation dose to the eyes could be reduced approximately by $30.4 \%$ with organ-based tube current modulation (14).

This study has several limitations. First, the study population was relatively small. Nonetheless, the statistical analysis reached the level of significance and relevant results could be obtained. Second, actual 3D brain volume was not measured due to a technical challenge related to segmentation quality and time resulting from a low contrast-to-noise ratio of the brain CT images. As previously mentioned, brain magnetic resonance imaging data is better than brain CT in quantifying actual 3D brain volume. However, the brain magnetic resonance examination is generally more expensive and takes more time than brain CT. Third, intracranial pressure data were not correlated with volumetric measures. However, pressure data is usually not available to radiologists during brain $\mathrm{CT}$ interpretation in patients with hydrocephalus. However, it will be interesting to investigate the implications of the correlation for managing patients with hydrocephalus in a future study. Finally, the interpretation of one experienced reader was used as the reference and inter-reader variability was not evaluated in this study. However, other reference standards are not available for ventricular and intracranial volumes. Furthermore, the experienced radiologist interpretation is the best among the currently available evaluations and a considerable inter-reader variability would complicate the statistical analysis.
In conclusion, the ventricular volume percentage quantified using 3D brain $\mathrm{CT}$ data is useful for interpreting serial changes in hydrocephalus.

\section{Conflicts of Interest}

The author has no potential conflicts of interest to disclose.

\section{ORCID iD}

Hyun Woo Goo

https://orcid.org/0000-0001-6861-5958

\section{REFERENCES}

1. Naidich TP, Epstein F, Lin JP, Kricheff II, Hochwald GM. Evaluation of pediatric hydrocephalus by computed tomography. Radiology 1976;119:337-345

2. Yabuuchi H, Kamitani T, Sagiyama K, Yamasaki Y, Matsuura Y, Hino $T$, et al. Clinical application of radiation dose reduction for head and neck CT. Eur J Radiol 2018;107:209-215

3. Sze RW, Ghioni V, Weinberger E, Seidel KD, Ellenbogen RG. Rapid computed tomography technique to measure ventricular volumes in the child with suspected ventriculoperitoneal shunt failure II: clinical application. J Comput Assist Tomogr 2003;27:668-673

4. Yamin G, Cheecharoen P, Goel G, Sung A, Li CQ, Chang YA, et al. Automated CT registration tool improves sensitivity to change in ventricular volume in patients with shunts and drains. Br J Radiol 2020;93:20190398

5. Wilk R, Kluczewska E, Syc B, Bajor G. Normative values for selected linear indices of the intracranial fluid spaces based on CT images of the head in children. Pol J Radiol 2011;76:16-25

6. Ragan DK, Cerqua J, Nash T, McKinstry RC, Shimony JS, Jones $\mathrm{BV}$, et al. The accuracy of linear indices of ventricular volume in pediatric hydrocephalus: technical note. $J$ Neurosurg Pediatr 2015;15:547-551

7. Mardini S, See LC, Lo LJ, Salgado CJ, Chen YR. Intracranial space, brain, and cerebrospinal fluid volume measurements obtained with the aid of three-dimensional computerized tomography in patients with and without Crouzon syndrome. J Neurosurg 2005;103(3 Suppl):238-246

8. Liu J, Huang S, Ihar V, Ambrosius W, Lee LC, Nowinski WL. Automatic model-guided segmentation of the human brain ventricular system from CT images. Acad Radiol 2010;17:718726

9. Multani JS, Oermann EK, Titano J, Mascitelli J, Nicol K, Feng R, et al. Quantitative computed tomography ventriculography for assessment and monitoring of hydrocephalus: a pilot study and description of method in subarachnoid hemorrhage. World Neurosurg 2017;104:136-141

10. Goo HW. Semiautomatic three-dimensional threshold-based cardiac computed tomography ventricular volumetry in 
repaired tetralogy of fallot: comparison with cardiac magnetic resonance imaging. Korean J Radiol 2019;20:102-113

11. Goo HW. Volumetric severity assessment of Ebstein anomaly using three-dimensional cardiac CT: a feasibility study. Cardiovasc Imaging Asia 2019;3:61-67

12. Yang DH, Goo HW. Pediatric 16-slice CT protocol: radiation dose and image quality. J Korean Radiol Soc 2008;59:333-347

13. Goo HW. CT radiation dose optimization and estimation: an update for radiologists. Korean J Radiol 2012;13:1-11

14. Greess H, Lutze J, Nömayr A, Wolf H, Hothorn T, Kalender WA, et al. Dose reduction in subsecond multislice spiral CT examination of children by online tube current modulation. Eur Radiol 2004;14:995-999

15. Wang J, Duan X, Christner JA, Leng S, Grant KL, McCollough $\mathrm{CH}$. Bismuth shielding, organ-based tube current modulation, and global reduction of tube current for dose reduction to the eye at head CT. Radiology 2012;262:191-198

16. Lee KB, Goo HW. Quantitative image quality and histogrambased evaluations of an iterative reconstruction algorithm at low-to-ultralow radiation dose levels: a phantom study in chest CT. Korean J Radiol 2018;19:119-129

17. Cho HH, Lee SM, You SK. Pediatric head computed tomography with advanced modeled iterative reconstruction: focus on image quality and reduction of radiation dose. Pediatr Radiol 2020;50:242-251

18. Huff TJ, Ludwig PE, Salazar D, Cramer JA. Fully automated intracranial ventricle segmentation on $\mathrm{CT}$ with $2 \mathrm{D}$ regional convolutional neural network to estimate ventricular volume. Int J Comput Assist Radiol Surg 2019;14:1923-1932

19. Klimont M, Flieger M, Rzeszutek J, Stachera J, Zakrzewska A, Jończyk-Potoczna K. Automated ventricular system segmentation in paediatric patients treated for hydrocephalus using deep learning methods. Biomed Res Int 2019;2019:3059170

20. Kamochi H, Sunaga A, Chi D, Asahi R, Nakagawa S, Mori $M$, et al. Growth curves for intracranial volume in normal Asian children fortify management of craniosynostosis. J Craniomaxillofac Surg 2017; 45:1842-1845

21. Patel DM, Tubbs RS, Pate G, Johnston JM Jr, Blount JP. Fastsequence MRI studies for surveillance imaging in pediatric hydrocephalus. J Neurosurg Pediatr 2014;13:440-447

22. Lee $\mathrm{E}, \mathrm{Goo} \mathrm{HW}$, Lee JY. Age- and gender-specific estimates of cumulative CT dose over 5 years using real radiation dose tracking data in children. Pediatr Radiol 2015;45:1282-1292 